\title{
PERCEIVED SERVICES AND REPORTED OPINIONS ABOUT SELF-CARE DURING PREGNANCY AND LACTATION BY COMMUNITY PHARMACISTS: A CROSS-SECTIONAL STUDY
}

\author{
SHRUTI S. SHIROMWAR* \\ Department of Pharmacology and Toxicology, Faculty of Pharmacy, Northern Border University, Rafha, Kingdom of Saudi Arabia. \\ Email: shruti_pharmacology@yahoo.com
}

Received: 12 May 2019; Revised and Accepted: 21 June 2019

\begin{abstract}
Objective: The present study was planned to evaluate the community pharmacists' services regarding self-care during pregnancy and lactating women in the Northern Border Region of Saudi Arabia.

Methods: A cross-sectional questionnaire-based survey was performed among the community pharmacists in the Northern Border Region of Saudi Arabia consists of randomly elected 156 pharmacies. The study purpose briefly described face-to-face to the pharmacist on duty to fill self-care questionnaire regarding services and opinions intended for pregnant and breastfeeding women.

Results: Pharmacists specified that they have sufficient knowledge to offer advice (47.82\%) and to solve medication and health-related problems $(39.13 \%)$ during pregnancy and lactation. The confidence level of pharmacists to give drug-related opinion and counseling to patients was $69.56 \%$ and $39.13 \%$, respectively. All the participated pharmacists' want continue education program and $47.82 \%$ of pharmacist advised pharmacy school training to improve/update the knowledge on the issue.
\end{abstract}

Conclusion: Pharmacists have different opinions regarding pregnancy and breastfeeding associated illness and also highlighted the need for interventions, including continuing professional development and revision of the undergraduate pharmacy curriculum.

Keywords: Community pharmacist, Services, Pregnancy, Breastfeeding, Northern border Region, Saudi Arabia.

(C) 2019 The Authors. Published by Innovare Academic Sciences Pvt Ltd. This is an open access article under the CC BY license (http://creativecommons. org/licenses/by/4. 0/) DOI: http://dx.doi.org/10.22159/ajpcr.2019.v12i7.34075

\section{INTRODUCTION}

Self-care is essential to maintain the good health which is self-initiated, deliberate act under our own control which includes lifestyle changes, dietary manifestations, natural supplements, diagnostics from home and use of nonprescription or over-the-counter (OTC) products for minor ailments [1]. Community pharmacists play an important role to assist and encourage self-care to the patients by the above interventions. Moreover, pharmacists assist patients for proper selection and use of OTC products. One of the components of self-care action is self-medication because the use of OTC medications for the major and minor symptoms for any illness is simply available in the community pharmacy without a prescription in many developing countries [2-4]. The occurrence of self-medication throughout the pregnancy and breastfeeding women was found to be in the range between $25 \%-68 \%$ and $17 \%-52 \%$, respectively [5-9]. Antibiotics, nonsteroidal anti-inflammatory drugs, antitussive, decongestant, antiviral drugs, anti-allergies, laxatives, antacids, vitamins, and herbal products, etc., are most common and easily available in the pharmacies without a prescription [10-12]. Nowadays, the use of these medications during pregnancy and lactation becomes a core element concerning to the pharmacist as well as for special population (pregnant and lactating woman's) due to the lack of professional guidance for appropriate and safe use of OTC medicines for the minor illness. The International Pharmaceutical Federation Council (2011) approved an article about the role of the pharmacist to deal with the special population which states guidelines on Good Pharmacy Practice to ameliorate the health of pregnant and lactating women [13]. Many studies are carried out to define the role of community pharmacists to deal with special population sickness in USA, Canada, Iceland, Kuwait, Norway, Thailand, Uganda, and Qatar [14-18]. Most of these studies evaluated the pharmacist's responses to treat symptoms observed during pregnancy and lactation to advice and counsel the special population along with their recommendations and knowledge about medications. But still, we need to accomplish studies on the services and perspectives of community pharmacists to deal with the pregnant and breastfeeding woman's especially in the developing countries were many drugs dispensed to the patients without the prescription.

Based on the above facts, a cross-sectional self-care questionnaire-based survey was carried out to evaluate the role of community pharmacist's services and their recommendation to tackle specific illness in special population in Northern Border region of Saudi Arabia.

\section{METHODS}

Study outline

The cross-sectional questionnaire-based survey was conducted among the community pharmacist of the Northern Border region of Saudi Arabia has an area of $111,797 \mathrm{~km}^{2}$ with the population of 320,524 (2010 census) as per the data stated by Saudi Geographical Society [19].

This survey-based study carried out within the period of 5 months from November 2018 to March 2019. Single employed register community pharmacist was included from 156 pharmacies from the different parts of the Northern Border Region. By expecting 98\% feedback through adequate and systemically organized random selection. The purpose of the survey was briefly explained to the pharmacist on duty (face-toface) to fill the self-care questionnaire (prepared in English and Arabic language) regarding services and opinions offered by a pharmacist for pregnant and lactating women. The pharmacist is free to decide whether to participate in the study or not. Those who are willing to be the part of the study were allowed to fill the self-care-based questionnaires and were collected from them secretly within 4-5 days. In this selfcare questionnaire-based survey, we used the modified authenticated questionnaires, which were used in the earlier studies conducted in Kuwait, Thailand, and France [18,20,21]. 
Questionnaires and components

Before the start of the actual study, the trial was carried out in five community pharmacists to observe if any modifications are needed to recover better and satisfactory results. Survey consist of four division first portion contains demographic and other characteristics information of registered pharmacist which covers gender, age in year, basic qualification in pharmacy, and the work experience, etc. Second portion comprised 8 inquiries to get the information about the services provided by the community pharmacist for self-care during pregnancy and lactation which includes the investigation of accessibility of educational pamphlet or brochure to boost the health of pregnant and breastfeeding women, experiences in offering services to pregnant and breastfeeding women along with their numbers visit pharmacies per week, the most frequently offered services by them for pregnant and breastfeeding women.

The third portion comprised the Pharmacists' responses to the specific 15 common symptoms in pregnancy and specific 12 common symptoms in breastfeeding, which are often faced by the pregnant and breastfeeding women. This involves pharmacist's recommendations for these symptoms when the pregnant and breastfeeding women visit a pharmacist for the advice. Here, pharmacist has to select any one option for each symptom like dispense medicine, refer to a doctor and provide any advice without dispensing medicine.

The last portion consists of five assertions to get the information about the pharmacists' opinions related to self-care in pregnancy and breastfeeding. Estimation of the response was carried out by three criteria such as agree, disagree, and neutral. Along with this, it includes questions to understand the pharmacists' opinion for the need of pharmacy school training and execution of continuing education programs to update the information about the self-care in pregnancy and breastfeeding. Assessments of preferred training methods (such as attending lecture, receiving regular newsletter or others) and most common source referred by the pharmacists (such as website, books, articles or journals) to deal with the special population for any illness.

\section{Statistical data analysis}

Obtained data were coded and analyzed using MS Excel 2010 program using descriptive statistics and inferential statistics with the representation of data by figures. Frequency and percentage calculated for discrete variables, maximum, minimum, and mean calculated for continuous variables.

\section{RESULTS}

One hundred and thirty-eight $(88.46 \%)$ community pharmacists from the total of 156 pharmacies were participated in the study, while $11.53 \%$ refused to take part due to the lack of time and some personal issues.

\section{Credentials and demographic information of participated pharmacists}

According to demographic investigations, $78.26 \%$ of registered community pharmacists were male while rests $21.73 \%$ were females. The $60.86 \%$ and $34.78 \%$ of register community pharmacists had aged in between $20-29$ years and 30-39 years, respectively, while $4.34 \%$ of community pharmacists have age above 40 years. Similarly, $52.17 \%$ of community pharmacists were bachelor in pharmacy and 39.13\% doctor in pharmacy, whereas $8.69 \%$ were master in pharmacy. Most of the community pharmacists, i.e., $82.60 \%$ have experienced more than 10 years (Fig. 1).

Details regarding availability of information, services experience, and services provided per week by community pharmacists for self-care in pregnancy and breastfeeding women

Fig. 2 explains about $73.91 \%$ of community pharmacists were apprehended with satisfactory, informative pamphlets or brochures to encourage and boost the health of pregnant and breastfeeding women. Most of the pharmacists got that information from drug companies, whereas remaining $26.08 \%$ of pharmacists were deficient for providing informative pamphlet or brochures. Around $60.86 \%$ and $52.17 \%$ of community pharmacists had experience in providing services to pregnant and breastfeeding women, respectively, while $34.78 \%$ of pharmacists had no experience in providing services to both types of populations. Services provided by the community pharmacists per week to the pregnant and breastfeeding women were found to be $39.13 \%$ and $17.39 \%$, respectively. Most of the community pharmacists revealed their interest in providing the advice to pregnant women regarding lifestyle changes like to follow a good and healthy diet, daily exercise along with pharmacist also recommended some vitamins and food supplements, while for breastfeeding women pharmacists instructed about weight control and guided them for the use of contraceptive devices.

Pharmacist's response for specific symptoms in pregnancy and breastfeeding women

Most of the time pregnant and breastfeeding women preferred to go to the pharmacy instead of going to hospitals for sought of medicine regarding minor illness such as in pregnant women with nausea and vomiting, indigestion, headache, back pain, respiratory problems, fever, constipation, diarrhea, and swelling of legs and feet. Similarly, in breastfeeding women with a sore or cracked nipple, engorgement, insufficient milk, mastitis, headache, respiratory tract infections, etc. All the responses were given by participated community pharmacists for these common and usually occurring in pregnant and breastfeeding women were analyzed by considering the three different directions given by pharmacist such as dispensing medicine for the treatment of the symptoms refer the patient directly to doctors and provide advice without dispensing any medicines.

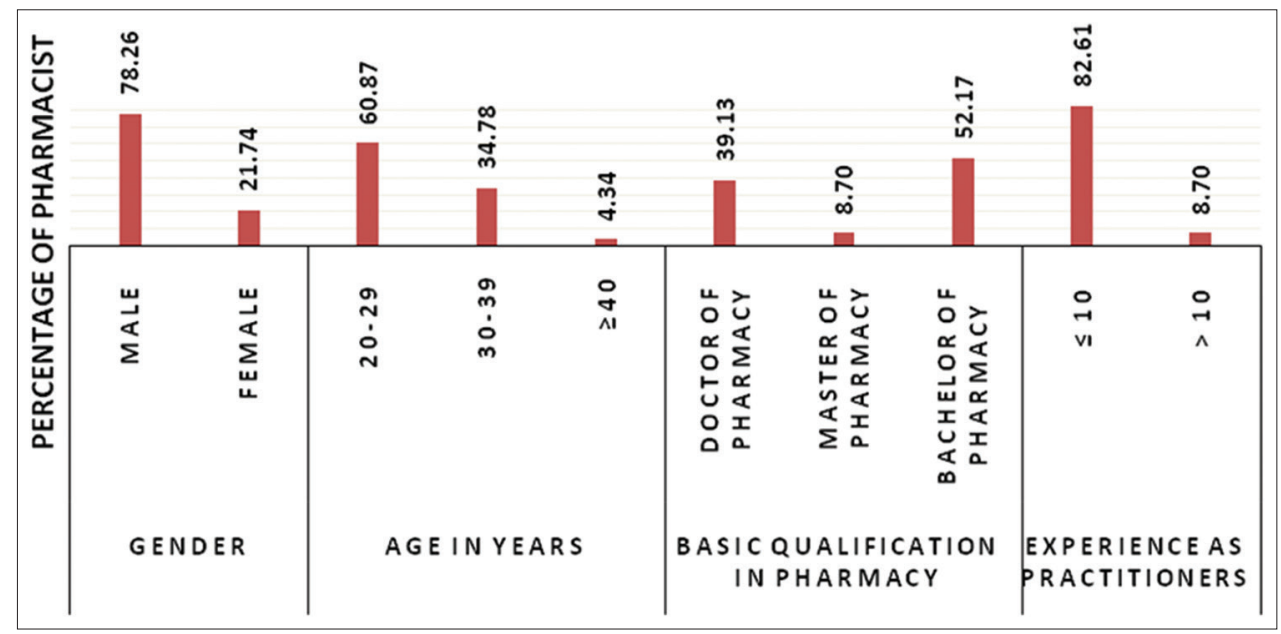

Fig. 1: Demographic and other characteristic information 
Fig. 3 represents the percentage of pharmacists responses for the treatment of 15 symptoms, which occurs commonly in pregnant women. About $95.65 \%$ of pharmacists suggested referring to a doctor without dispensing any medicines to the pregnant women with the ailments such as vaginal itching and simple discharge, hemorrhoids, and swelling feet and legs, while for other symptoms such as indigestion (69.56\%), back pain (78.26\%), insomnia (86.95\%), fever and ache $(73.92 \%)$, constipation $(60.86 \%)$, and diarrhea $(91.3 \%)$ pharmacists referred patients to physicians. Less than $50 \%$ of pharmacists dispensed medicines for nausea and vomiting, respiratory problems such as dry and productive cough, and runny nose but in case of headache, $52.17 \%$ of pharmacists dispensed medicines to pregnant women. Less than $10 \%$ of pharmacists dispensed medicines to minor illness like indigestion, back pain, and diarrhea. Less than $20 \%$ of pharmacists provided advice for all the symptoms except in case of indigestion, i.e., $21.73 \%$.

Details of community pharmacists' responses to the specific symptoms in breastfeeding women

Fig. 4 represents the details of community pharmacists' responses to the 12 specific common and frequently occurring symptoms in breastfeeding women. The breastfeeding women with the problem like mastitis, i.e., inflammation of breast tissue were directly referred to physicians by all pharmacists. The condition such as breast tissue overfills with milk, blood, and other fluids (engorgement), 95.65\% of pharmacists referred the patient to doctor, while in other cases such as sore and cracked nipple (56.52\%), insufficient milk (73.43\%), diarrhea $(73.91 \%)$, constipation $(60.86 \%)$, hemorrhoids $(86.95 \%)$, headache (34.78\%), sore throat (65.21\%), runny nose (52.17\%), dry cough $(47.82 \%)$, and productive cough $(52.17 \%)$ pharmacists referred patients to doctor. In the case of engorgement and mastitis, nobody dispensed medicines without the prescription. In case of headache $56.52 \%$ and runny nose, $43.47 \%$ of pharmacists dispensed medicines without a prescription while less than $40 \%$ of them have dispensed medicine for other symptoms. Not everyone pharmacist provided any advice in case of mastitis, but for insufficient milk and constipation $17.39 \%$ while in other conditions, less than $10 \%$ of community pharmacists were interested to provide advice to the patients.

Community pharmacists' responses for eight common symptoms in pregnancy and breastfeeding women

Fig. 5 shows the direction of pharmacists for the eight common symptoms which occurs in pregnancy and breastfeeding women. Most of the pharmacists dispensed medicines or referred to the physicians rather than providing only advice without dispensing any medicines for the treatment of these common symptoms which occurs in both the population. More than half of the participants recommended medications for the treatment of headache in both pregnant and breastfeeding women. While in relation to all symptoms, most of the pharmacists referred patients to doctors except in headache, i.e., $30.34 \%$ and $34.78 \%$ in pregnancy and breastfeeding women's, respectively. Less than $20 \%$ of pharmacists provided advice for the common symptoms in both cases. In the case of hemorrhoids, $<5 \%$ of pharmacists dispensed drugs to the patient without prescription in breastfeeding women's while in pregnancy, nobody dispensed any medication.

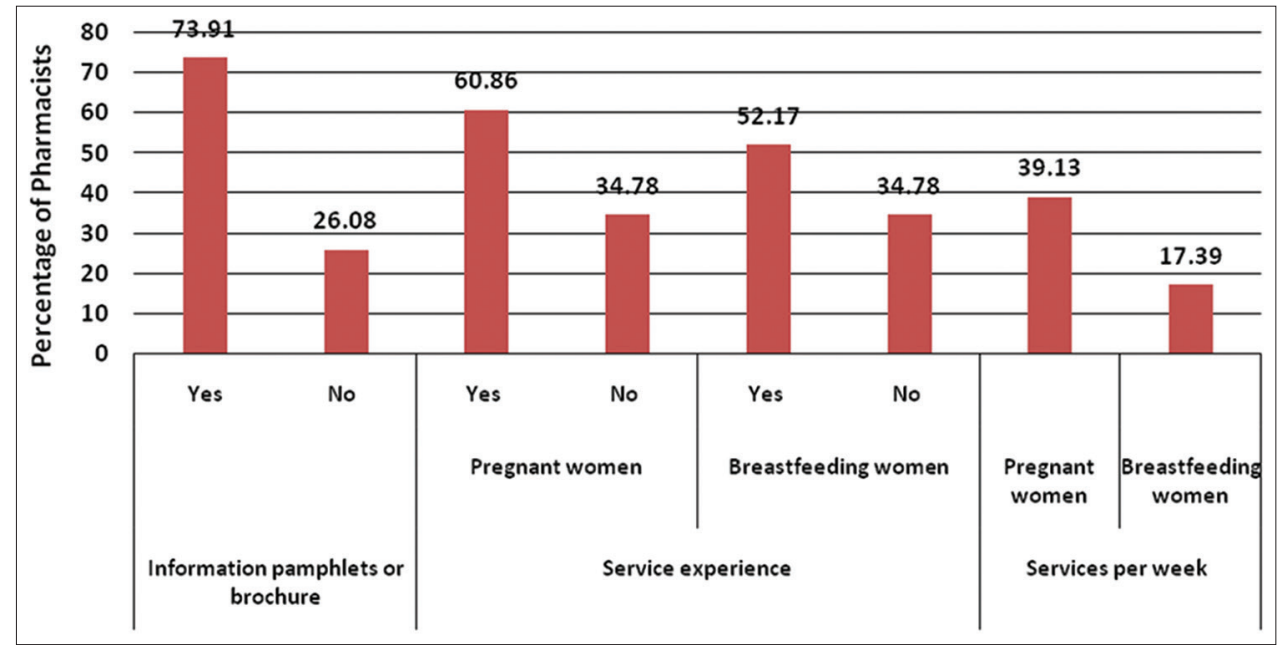

Fig. 2: Details of information availability and services experience and services provided per week by community pharmacists for self-care in pregnancy and breastfeeding women

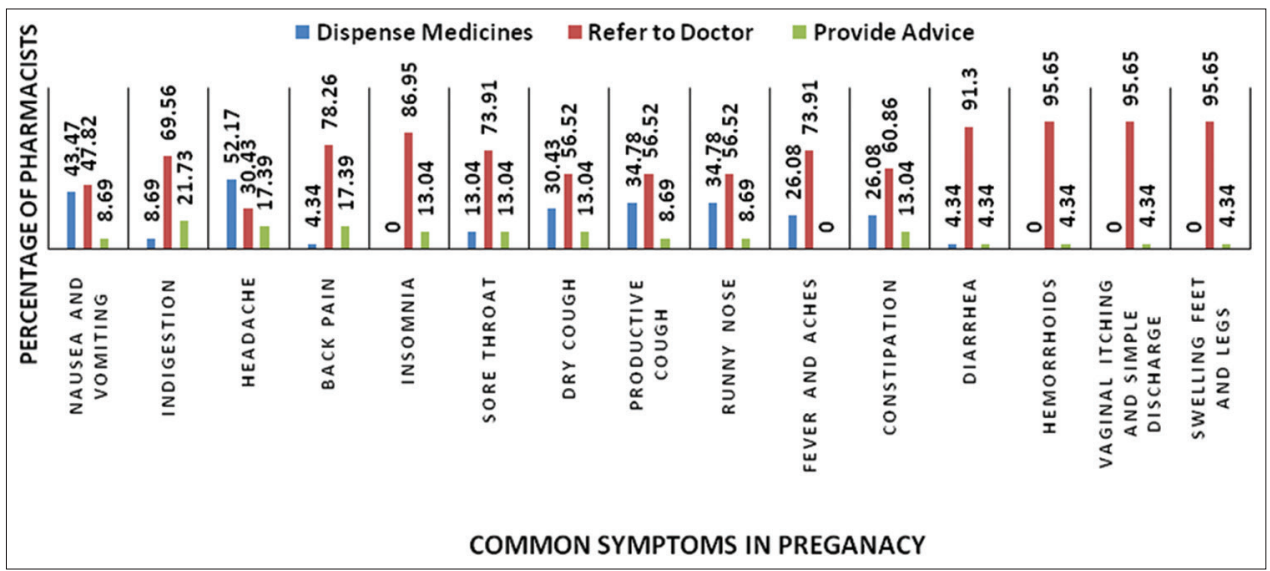

Fig. 3: Details of community pharmacists' responses for specific symptoms in pregnancy 
Community pharmacists' opinions about self-care in pregnancy and breastfeeding women

The first assertion about self-care provision, $65.21 \%$ pharmacists agreed that they are well qualified to offer advice and an OTC therapy to treat symptoms in pregnant and breastfeeding women. Opinion in case of recommending OTC therapy and counseling 39.13\% and $52.17 \%$ of pharmacists were agreed in pregnancy and breastfeeding, respectively, while $21.73 \%$ of pharmacists were neutral, they did not have any opinion. More than $50 \%$ of pharmacists disagreed while $34.78 \%$ were neutral to give any opinion for the use of OTC medication as they are not safe in both the population. About $73.91 \%$ and $43.47 \%$ of pharmacists were confident to advice and counsel the pregnant and lactating women's respectively and $13.04 \%$ disagreed for that in both pregnant and breastfeeding women. Less than half of pharmacists agreed that they have satisfactory knowledge to resolve the medication and health-care problems in pregnant $(47.82 \%)$ and breastfeeding women's (39.13\%) while $43.78 \%$ of pharmacists were neutral, i.e., neither agreed nor disagreed (Fig. 6).

Opinion on educational programs regarding self-care in pregnant and breastfeeding women

Fig. 7 represents the opinion of community pharmacists regarding the need for education for self-care in pregnant and breastfeeding women. All (100\%) pharmacist were agreed that continuing education program regarding this topic would be of worth and significant for their practice.

Introducing the pharmacy school training for the pharmacists to get the knowledge, information, recent advance techniques, and medicines for the handling of the special population around $47.82 \%$ of pharmacists were agreed whereas $21.73 \%$ of pharmacists disagreed whereas $30.43 \%$ of pharmacists had a neutral opinion.

About $60.86 \%$ of pharmacists chose to attain the lecture as a mode of training because it is the most appropriate method whereas around $34.78 \%$ of pharmacists wanted to receive regular newsletter for the training purpose on this topic so as to be updated with new information's that will be helpful for them without disturbing their working hours.

According to the community pharmacists, $43.47 \%$ of them usually refers books to overcome the sign and symptoms frequently occurs in pregnant and breastfeeding women's while $30.43 \%$ refers websites and $21.73 \%$ of pharmacists use journal articles to enhance their knowledge, confidence and also to provide better and efficient services to pregnant and breastfeeding women.

Data were analyzed using MS Excel 2010 program using descriptive statistics and inferential statistics. Frequency and percentage were calculated for discrete variables. Maximum, minimum, and mean were calculated for continuous variables.

\section{DISCUSSION}

The given study represents that the pregnant and breastfeeding woman from Northern Border region of Saudi Arabia habitually visit a pharmacy for the medicines, guidance to treat the common as well as most frequently occurring symptoms and for other health-

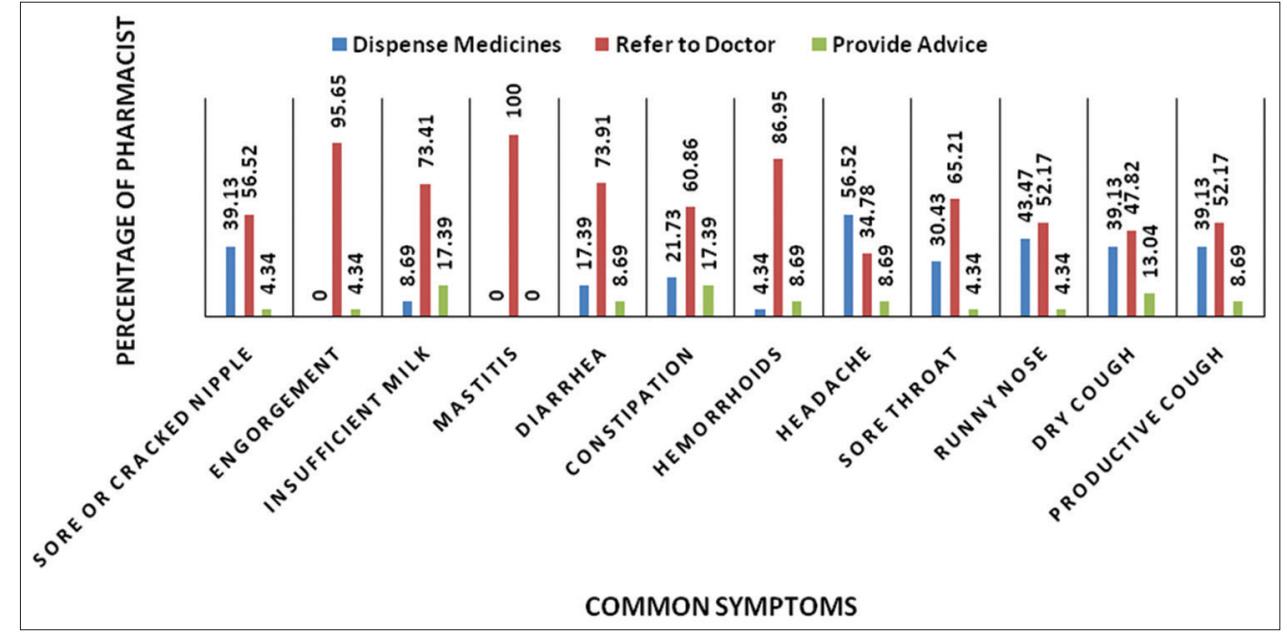

Fig. 4: Details of community pharmacists' responses to the specific symptoms in breastfeeding women

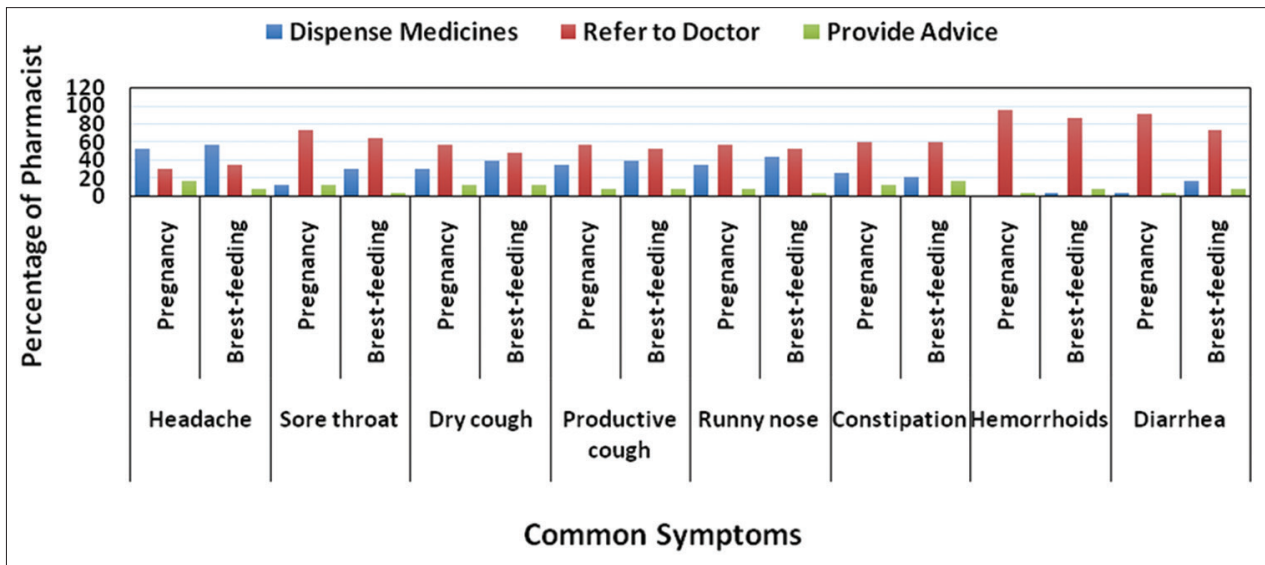

Fig. 5: Community pharmacists' responses for eight common symptoms in pregnancy and breastfeeding women 


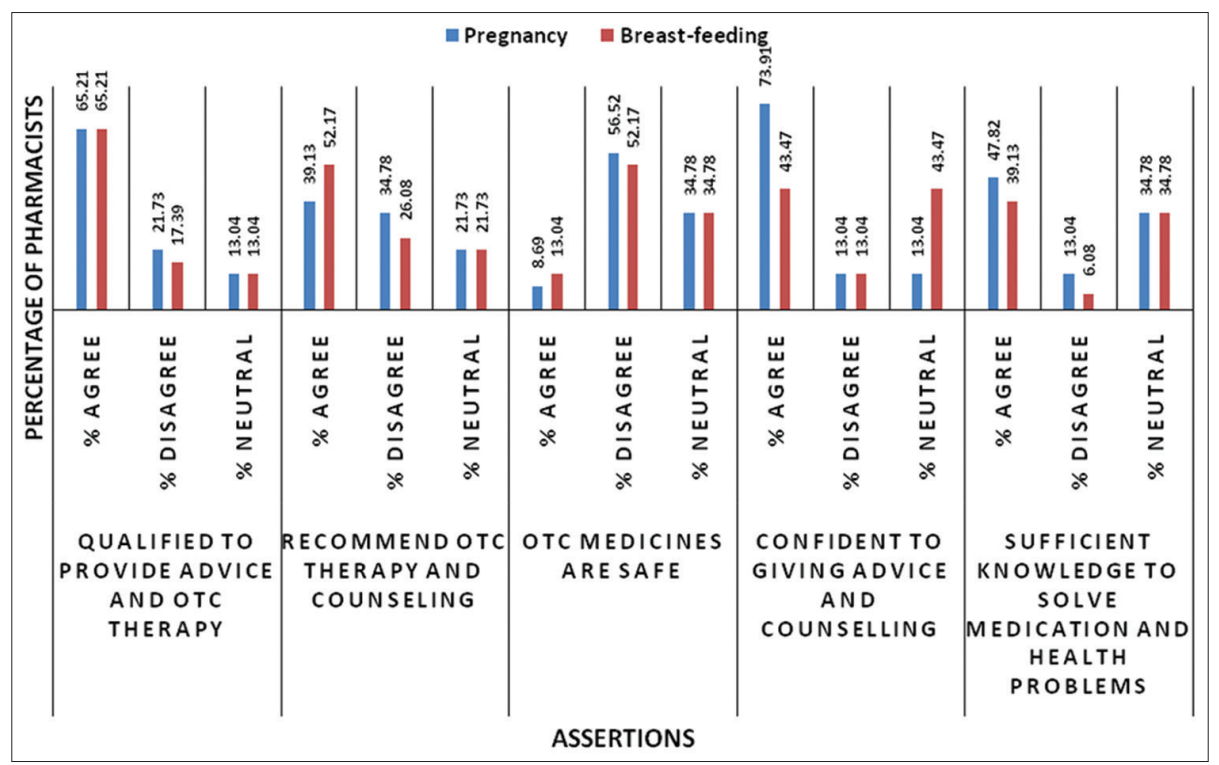

Fig. 6: Community pharmacist's opinion about assertions for self-care in pregnant and breastfeeding women

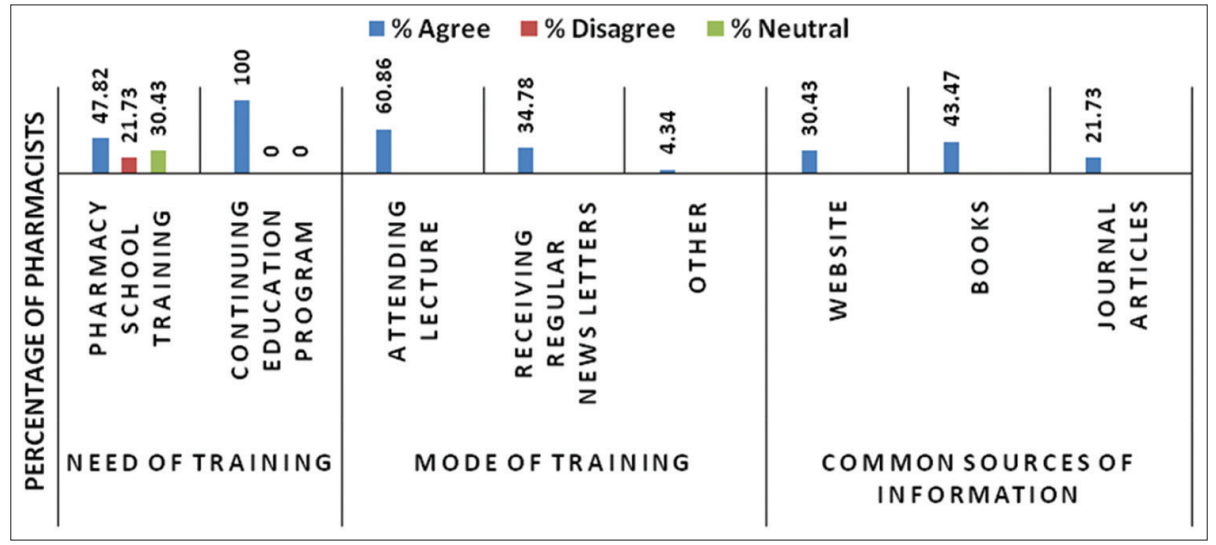

Fig. 7: Details of opinion on educational programs regarding self-care in pregnant and breastfeeding women

related problems. A highly skilled, confident with the comprehensive knowledge register community pharmacists are needed for satisfying above needs of pregnant and lactating women. However, in this study, we found that still the community pharmacists were deprived of all these qualities because most of the time they referred the patient to the doctors instead of giving a piece of advice or dispensing any safe medicines for the common complaints regarding patient health.

The undertaken study provides elementary quantitative data which support the limited amount of existing literature regarding the services provided by community pharmacists to pregnant and lactating women in emerging countries. It will help for an evaluation of existing pharmacy practices toward self-care during the pregnancy and lactation. Furthermore, it will provide awareness about the future aids for the progress of community pharmacist to distribute proper medicines, adequate information and to solve complications of pregnant and lactating women sickness in Northern Border region of Saudi Arabia.

As per our knowledge, this is the first study conducted in Saudi Arabia and second from the entire Gulf countries. In the given study, we discovered that community pharmacist frequently provided the common services to pregnant and lactating women like the advice about suitable behavior such as to follow a good lifestyle, daily exercise. They also offered them (special population) some vitamins and food supplements, advice for contraceptive devices, and dispensed medicines which remain safe in pregnant and lactating mother.

Our study results specify some similarity with the study conducted previously in Kuwait [18] except in some condition such as in dispensing a drug to treat fever and ache, vaginal itching and simple discharge, hemorrhoids, and swelling of foot and legs in pregnancy while insufficient milk production in breastfeeding women. The community pharmacist specified that generally, people with symptoms such as headache, respiratory problems, GI related problems during pregnancy and lactation wish to consult them instead of going to the physician except in conditions like hemorrhoids for that most of the pharmacist refer patient to doctors. This occurs mainly due to freely computable pharmacies and it is perceived that the community pharmacists are standard and acknowledged as extreme convenient health-care person to whom a significant number of populations consult for the various opinions and to get the many services from them [22]. Furthermore, the pharmacist can give sufficient time to discuss patients' problems instead of a doctor for the non-serious ailment [23-25].

All the findings of the study show that health professionals should reflect as constancy between safe traditional theories and modern health systems to safeguard the best self-care practices for both women and their babies. For this purpose, the pharmacist should have sufficient information and accountable context for dealing 
with women who need their advice or services to improve maternal health. Here, in the study, the participated community pharmacist also requested to give an answer regarding the services and opinion they want to indicate for pregnancy and lactation associated problems. Most of the time, pharmacist specified the medicines for the headache and nausea and vomiting, running nose as well as sore and cracked nipple.

In contrast, an earlier study carried out in France discovered that medications were regularly suggested by the pharmacists for pain, fever, nose and oropharynx disorders, venous insufficiency, dyspepsia, and constipation [20]. In Thailand, about $75 \%$ of pharmacists offered medication for the treatment of headache, runny nose, and sore throat [21]. A recent study in Kuwait described that more than 50\% of community pharmacists dispensed medicines to treat headache, respiratory tract problems, constipation, indigestion, sore and crack nipple, insufficient milk, etc., during pregnancy and lactation [18], while community pharmacists from Norway proposed that more than $62 \%$ of non-pharmacological therapy as well as referrals to a doctor's [26].

All these studies explained the huge variance in a working practice of the community pharmacist from the different countries concerning the services and advice for the treatment of pregnancy and breastfeeding associated illness. Possibly this occurs because of the difference in the governing environment, study pattern of an undergraduate program, easy accessibility of remedial products at pharmacies, etc. Less than $20 \%$ of pharmacists provided advice for most of the symptoms which emphasize that pharmacist required to have sufficient knowledge, information, and evidence regarding the self-care practices which plays a key role to improve specific and common illness without the use of medicines. Always it is important that the community pharmacist should be updated with sufficient information to get the correct assumption regarding risk versus benefit ratio to treat the pregnant and lactating women's by systemic and effective to advise as well as counseling to circumvent incompetence of community pharmacist for providing sufficient authentic data about the practice of medicines during pregnancy and lactation.

The undertaken study validates that the pharmacists have diverse knowledge levels to solve the medication and health-related problems in the pregnancy and breastfeeding related illness. Therefore, they required continuing professional development and a revision of the undergraduate pharmacy curriculum to overcome the knowledge gaps between pharmacy students and practitioners in maternal-fetal drug usage. This will help the community pharmacists to distribute an appropriate medicine and care to pregnant and lactating women. The study outcomes and whatever the end results we got showed the requirement for further qualitative research to describe and understand these specialists.

\section{CONCLUSION}

The present study findings revealed that community pharmacists in the Northern Border Region of Saudi Arabia discloses the different recommendations pattern for the treatment of pregnancy as well as breastfeeding associated illnesses. The participated community pharmacists suggested updating their knowledge by continuing professional education and proposed to modify and evaluate the undergraduate pharmacy curriculum to diminish the gap in between the pharmacy graduates and pharmacy practitioners for effective dispensing of medicines to the special population.

\section{ACKNOWLEDGMENT}

The author would like to thanks the entire registered community pharmacists who were participated in the survey and gave their important opinion to improve the knowledge to deal with the special population.

\section{AUTHORS' CONTRIBUTION}

Ms. Shruti has designed the research proposal and she has also revised and wrote the manuscript.

\section{CONFLICTS OF INTEREST}

Author does not have any conflicts of interest.

\section{REFERENCES}

1. Segall A, Goldstein J. Exploring the correlates of self-provided health care behaviour. Soc Sci Med 1989;29:153-61.

2. Geissler PW, Nokes K, Prince RJ, Odhiambo RA, Aagaard-Hansen J, Ouma JH, et al. Children and medicines: Self-treatment of common illnesses among Luo schoolchildren in Western Kenya. Soc Sci Med 2000;50:1771-83.

3. Pranav V, Narayanan P, Guddattu V. Self-medication practice among urban slum dwellers in Udupi taluk, Karnataka, India. Int J Pharm Pharm Sci 2017:9:19-23.

4. Saharan VD, Pandey MS. A study of prevalence of self medication practice among people of Mumbai. Int J Pharm Pharm Sci 2015;7:253-6.

5. Ebrahimi H, Atashsokhan G, Amanpour F, Hamidzadeh A. Selfmedication and its risk factors among women before and during pregnancy. Pan Afr Med J 2017;27:183.

6. Araújo DD, Leal MM, Santos EJ, Leal LB. Consumption of medicines in high-risk pregnancy: Evaluation of determinants related to the use of prescription drugs and self-medication. Braz J Pharm Sci 2013;49:491-9.

7. Nakamura MU, Junior LK, Pasquale M. Uso de fármacosnagravidez: Benefício e custo. Rev Bras Ginecol Obstet 2008;30:1-4.

8. Chiriboga CA. Fetal alcohol and drug effects. Neurologist 2003;9:267-79.

9. Szymanowski K, Chmaj-Wierzchowska K, Florek E, Opala T. Influence of tobacco smoking to development of the fetus, newborn and child: A review. Przegladlekarski 2006;63:1135-7.

10. Kim HJ, Yang YM, Choi EJ. Use patterns of over-the-counter (OTC) medications and perspectives on OTC medications among Korean adult patients with chronic diseases: Gender and age differences. Patient Prefer Adherence 2018;12:1597-606.

11. Sansgiry SS, Bhansali AH, Bapat SS, Xu Q. Abuse of over-thecounter medicines: A pharmacist's perspective. Integr Pharm Res Pract 2017;6:1-6.

12. Abduelkarem AR, Mustafa H. Use of over-the-counter medication among pregnant women in Sharjah, United Arab Emirates. J Pregnancy 2017;2017:4503793.

13. International Pharmaceutical Federation. FIP Reference Paper on the Effective Utilization of Pharmacists in Improving Maternal, Newborn and Child Health (MNCH). FIP Council, September 2011. Available from: https://www.fip.org/www/uploads/database_file. php?id=325\&table id. [Last accessed on 2019 Apr 14].

14. Leung HY, Saini B, Ritchie HE. Medications and pregnancy: The role of community pharmacists a descriptive study. PLoS One 2018; 13:e0195101.

15. Schrempp S, Ryan-Haddad A, Gait KA. Pharmacist counseling of pregnant or lactating women. J Am Pharm Assoc (Wash) 2001;41:887-90

16. Samuel N, Einarson A. Medication management during pregnancy: Role of the pharmacist. Int J Clin Pharm 2011;33:882-5.

17. Ronai C, Taylor JS, Dugan E, Feller E. The identifying and counseling of breastfeeding women by pharmacists. Breastfeed Med 2009;4:91-5.

18. Albassam A, Awad A. Community pharmacists' services for women during pregnancy and breast feeding in Kuwait: A cross-sectional study. BMJ Open 2018;8:e018980.

19. Saudi Geographical Society. Available from: http://www.saudigs.org/ en. [Last accessed on 2019 Apr 14].

20. Damase-Michel C, Vié C, Lacroix I, Lapeyre-Mestre M, Montastruc JL. Drug counselling in pregnancy: An opinion survey of French community pharmacists. Pharmacoepidemiol Drug Saf 2004;13:711-5.

21. Sathon B. Self-care in Pregnancy and Breastfeeding: Views of Women and Community Pharmacists in Thailand. Dissertation Nottingham: University of Nottingham. 2010. Available from: http://www.eprints. nottingham. ac. uk/ 11605. [Last accessed on 2019 Apr 14].

22. Sadek MM, Elnour AA, Al Kalbani NM, Bhagavathula AS, Baraka MA, Aziz AM, Shehab A. Community pharmacy and the extended community pharmacist practice roles: The UAE experiences. Saudi Pharm J 2016;24:563-70. 
23. Kelly DV, Young S, Phillips L, Clark D. Patient attitudes regarding the role of the pharmacist and interest in expanded pharmacist services. Can Pharm J (Ott) 2014; 147:239-47.

24. Ayele AA, Mekuria AB, Tegegn HG, Gebresillassie BM, Mekonnen AB, Erku DA, et al. Management of minor ailments in a community pharmacy setting: Findings from simulated visits and qualitative study in Gondar town, Ethiopia. PLoS One 2018;13:e0190583.
25. Hammond T, Clatworthy J, Horne R. Patients' use of GPs and community pharmacists in minor illness: A cross-sectional questionnaire-based study. Fam Pract 2004;21:146-9.

26. Odalović M, Milanković S, Holst L, Nordeng H, Heitmann K, Tasić L, et al. Pharmacists counselling of pregnant women: Webbased, comparative study between Serbia and Norway. Midwifery 2016;40:79-86. 